\title{
A three-dimensional macroscopic fundamental diagram for mixed bi-modal urban networks
}

\author{
Nikolas Geroliminis a , Nan Zheng ${ }^{\mathrm{a}, *}$, Konstantinos Ampountolas ${ }^{\mathrm{a}, \mathrm{b}}$ \\ ${ }^{a}$ Urban Transport Systems Laboratory, École Polytechnique Fédérale de Lausanne, 1015 Lausanne, Switzerland \\ ${ }^{\mathrm{b}}$ School of Engineering, University of Glasgow, Glasgow G12 8LT, United Kingdom
}

\section{A R T I C L E I N F O}

\section{Article history:}

Received 20 November 2013

Received in revised form 23 February 2014

Accepted 10 March 2014

\section{Keywords:}

Bi-modal traffic

Macroscopic fundamental diagram

Passengers

Public transport

Clustering

\begin{abstract}
A B S T R A C T
Recent research has studied the existence and the properties of a macroscopic fundamental diagram (MFD) for large urban networks. The MFD should not be universally expected as high scatter or hysteresis might appear for some type of networks, like heterogeneous networks or freeways. In this paper, we investigate if aggregated relationships can describe the performance of urban bi-modal networks with buses and cars sharing the same road infrastructure and identify how this performance is influenced by the interactions between modes and the effect of bus stops. Based on simulation data, we develop a three-dimensional vehicle MFD (3D- $v$ MFD) relating the accumulation of cars and buses, and the total circulating vehicle flow in the network. This relation experiences low scatter and can be approximated by an exponential-family function. We also propose a parsimonious model to estimate a three-dimensional passenger MFD (3D-pMFD), which provides a different perspective of the flow characteristics in bi-modal networks, by considering that buses carry more passengers. We also show that a constant Bus-Car Unit (BCU) equivalent value cannot describe the influence of buses in the system as congestion develops. We then integrate a partitioning algorithm to cluster the network into a small number of regions with similar mode composition and level of congestion. Our results show that partitioning unveils important traffic properties of flow heterogeneity in the studied network. Interactions between buses and cars are different in the partitioned regions due to higher density of buses. Building on these results, various traffic management strategies in bi-modal multi-region urban networks can then be integrated, such as redistribution of urban space among different modes, perimeter signal control with preferential treatment of buses and bus priority.
\end{abstract}

(c) 2014 Elsevier Ltd. All rights reserved.

\section{Introduction}

Transportation systems in real cities are complex with many modes of transport sharing and competing for limited road infrastructure. While there is a vast literature of the dynamics and the modeling of congestion for vehicular traffic, the effect of service stops (like when a bus or taxi stops to board and alight passengers) in the overall performance of a large-scale urban system still remains a challenge. It is intuitive that the effect of these stops in the network capacity is almost negligible during light demand conditions, but nowadays city centers are experiencing high level of congestion and the frequency in

\footnotetext{
* Corresponding author. Tel.: +412169 32481; fax: +41216935060.

E-mail addresses: nikolas.geroliminis@epfl.ch (N. Geroliminis),
} 
time and space of these stops is significantly high. While one bus traditionally creates more congestion than a moving car, the additional delay per passenger carrying is smaller for large bus occupancies, making buses a more sustainable mode of transport compared to cars.

To understand the physics of urban mobility, traffic dynamics of multimodal urban networks need to be analyzed under various network structures. As multiple modes compete for limited urban space, conflicts and interactions are developed resulting in congestion. Existing literature on multimodal traffic mainly focuses on the design and operation of special bus lanes (Site and Filippi, 1998; Daganzo and Cassidy, 2008; Li et al., 2010; Tirachini and Hensher, 2011) or optimization of traffic signals with transit priority (Eichler and Daganzo, 2006; Mesbah and Currie, 2011; Christofa et al., 2013). However there is no significant body of work dedicated on the modeling of traffic dynamics and the influence of each mode on the network performance. Most of the existing works fall short either in the scale of application or the treatment of congestion dynamics (small scale and/or static models). For example, the work by Li et al. (2010) estimates traffic state for each mode based on a BPR (Bureau of Public Roads, 1964) type of model, which works only for static conditions. Site and Filippi (1998), Daganzo and Cassidy (2008) and Tuerprasert and Aswakul (2010) utilize the link-scale Fundamental Diagram, which can experience high scatter and therefore cannot provide accurate estimates of speed and travel time at the network level. These somehow detailed models can also be computationally complex when applied at the network scale.

An aggregated modeling for multimodal systems, following the concept of a macroscopic fundamental diagram (MFD), can be a strong alternative if it unveils similar properties as in the single-mode case of vehicular traffic. The idea of an MFD for car-only urban networks was initially proposed by Godfrey (1969) and was re-initiated later in Mahmassani et al. (1987) and Daganzo (2007). The demonstration of its existence with dynamic features and empirical data from Yokohama was recent by Geroliminis and Daganzo (2008). This work showed that the MFD is a property of the network itself (infrastructure and control) if the network is homogeneously congested and not very sensitive to demand, i.e. the spacemean flow is maximum for the same value of critical density of vehicles, for many origin-destination tables. Recent works also highlighted that the spatial distribution of congestion can affect the shape and the scatter of the MFD with higher flows observed for less spatial heterogeneity, e.g. Mazloumian et al. (2010), Geroliminis and Sun (2011), Mahmassani et al. (2013), and Knoop et al. (2013). Analytical theories have been derived for the shape of the MFD as a function of network and intersection parameters, using variational theory for homogeneous and heterogeneous network topologies, respectively (Daganzo and Geroliminis, 2008; Geroliminis and Boyaci, 2012; Leclercq and Geroliminis, 2013). The properties of a welldefined MFD, stability and scatter phenomenon are analyzed through many other simulation studies and experimental tests, such as Buisson and Ladier (2009), Ji et al. (2010), Gayah and Daganzo (2011), Saberi and Mahmassani (2012), and Ji and Geroliminis (2012), Mahmassani et al. (2013). Given the MFD of a network, effective traffic management strategies can be readily developed to mitigate congestion, examples including perimeter flow control in Keyvan-Ekbatani et al. (2012), Haddad et al. (2013) and Aboudolas and Geroliminis (2013) and cordon-based pricing in Zheng et al. (2012). Besides, Gonzales and Daganzo (2012) examined system optimum solutions for a transport system with cars and public transit share spaces for the morning commute problem. The authors solved user and system optimum for the bottleneck model and system optimum for the network model (with an MFD representation).

Building on the knowledge of the single-mode MFD, developing and understanding the dynamics of multimodal networks is promising. In this work, we seek to extend the modeling and the application of the single-mode MFD to a bi-modal (bus and cars) one, with the consideration of passenger flows and traffic performance of each mode. If this type of models holds for further scrutiny in multimodal networks, then network level control strategies can be developed to maximize urban mobility. The dynamics of traffic flow in bi-modal networks are more complicated due to the operational characteristics of buses and the interactions between cars and buses. Despite of this complication, simulation studies on small networks showed that a classical MFD applies (under certain conditions) for bi-modal urban networks as well, see e.g. Gonzales et al. (2011) and Zheng and Geroliminis (2013). However, the influence of each mode in the network dynamics and performance is still missing. This relationship, if known, will facilitate the development of control strategies at various levels, e.g. network bus priority (Christofa et al., 2013) or redistribution of urban space (Zheng and Geroliminis, 2013). Therefore we aim at investigating the relationship among the accumulation of cars and buses, and the traffic throughput or circulating flow of a network. Buses have scheduled service stops in addition to stops caused by traffic and signals, which makes the speed and the flow rate of buses lower than cars. On the other hand, buses carry significantly higher number of passengers. Therefore it is insufficient to simply consider vehicle flows and develop the relevant management strategies for a multimodal network. Zheng et al. (2013) presented initial simulation findings on a three-dimensional macroscopic relation between flow and accumulations for a bi-modal network, and proposed to develop a macroscopic passenger flow model.

A semi-analytical approach building on variational theory was developed to estimate an MFD for networks with cars and buses (Boyaci and Geroliminis, 2011). Buses influence traffic when they stop for passengers to enter or exit at bus stops. These stops were treated as periodic red phases of fictitious traffic signals that decrease the capacity of the road at the bus stop locations. While such a framework can provide important insights for network capacity for different operational characteristics of buses, it cannot directly integrate the heterogeneity in the spatial distribution of buses and cars in the network. To shed some light towards this direction a detailed micro-simulation of a congested bi-modal network is performed for various demand scenarios and a relationship between network flow and accumulations of cars and buses is investigated in the current work.

Both the vehicle flow and the passenger flow dynamics were not studied in detail and have to be further investigated. Furthermore, the ratio of car and bus density is not homogeneously distributed in urban networks (due to different location 
of bus lanes and service frequencies) and it is insufficient to assume a homogeneous interaction between the two modes for whole network. Thus, multimodal networks with significant presence of buses might experience different aggregated dynamics than car-only networks and might not follow an aggregated law similar to the MFD. In this case, network partitioning algorithms, such as the one presented in Ji and Geroliminis (2012) for car-only networks, can be applied to capture the spatial heterogeneity in flow characteristics.

The remaining part of the paper is organized as follows: Section 2 investigates the existence and the properties of a three-dimensional vehicular MFD (3D-vMFD) relating the accumulation of cars and buses, and the total circulating vehicle flow in the network based on simulation data with mixed traffic of cars and buses. Then a three-dimensional passenger MFD (3D-pMFD) is derived relating the accumulation of cars and buses and the passenger flow in a bi-modal network. Section 3 introduces an analytical approach to approximate the shape of the bi-modal passenger MFD, given the 3D- $v$ MFD. Afterwards, we investigate how partitioning can contribute to the modeling of heterogeneous networks. The last section summarizes the findings and discusses future research directions.

\section{A city-scale three-dimensional MFD for bi-modal traffic}

In this section, we investigate the relation among accumulation of cars and buses and circulating flow (both vehicle and passenger) in bi-modal traffic networks via simulation experiments. We show that an area of Downtown San Francisco exhibits a city-scale three-dimensional vehicle MFD (3D-vMFD) relating the accumulation of cars and buses to flow with moderate scatter under different bi-modal demand patterns. We further show that our test site exhibits a city-scale three-dimensional passenger MFD (3D-pMFD) relating accumulation of cars and buses, to the passenger flows in the bi-modal network.

The test site is a $3 \mathrm{~km}^{2}$ area of Downtown San Francisco including about 100 intersections and 430 links of total 100 lane-kms. The number of lanes varies from 2 to 5 lanes and the free flow speed is around $45 \mathrm{~km} / \mathrm{h}$. Traffic signals are all multiphase fixed-time operating on a common cycle length of $100 \mathrm{~s}$ for the west boundary of the area and $60 \mathrm{~s}$ for the rest. There is no any preferential treatment of buses, e.g. transit signal priority (TSP). Bus stops are in-lane of traffic and create queues of cars during stopping. The traffic flow in the (bi-modal) network comprises two vehicle classes, i.e. passenger cars and buses. Bus routes and frequencies for lines in the studied network have been obtained from the San Francisco Municipal Transportation Agency (SFMTA). Bus stops are influencing traffic as in most cases there are on-street with a capacity for one or two buses to serve passengers. Cars and buses are sharing the same infrastructure and operating in the same road lanes except in Section 2.5, where a preliminary investigation of dedicated bus lanes is carried out.

\subsection{A three-dimensional MFD for vehicle flows (3D-vMFD)}

Let us denote by $n_{c}$ the accumulation of cars and $n_{b}$ the accumulation of buses at a specific time interval, $Q_{m}$ is the total network circulating flow for each mode respectively, $m \epsilon\{b, c\}$ and $Q$ the total network flow (in vehicle per unit time), which is the sum of car and bus circulating flows, all estimated during the same interval. $Q_{m}$ is defined in accordance to Edie's definitions as $Q_{m}=\sum_{i} l_{i} q_{i m} / L$, where $l_{i}$ is the length of link $i, q_{i m}$ is the flow of link $i$ for mode $m$ and $L$ is the average link length (Edie, 1963). For the developed model, the flow $Q$ in the bi-modal network is considered to be a function of $n_{c}$ and $n_{b}$ given by (time interval $t$ is omitted from the equations for simplicity)

$$
Q=Q\left(n_{c}, n_{b}\right)=Q_{c}\left(n_{c}, n_{b}\right)+Q_{b}\left(n_{c}, n_{b}\right)
$$

To obtain the shape of (1) we perform extensive simulation experiments in the test network with time-dependent asymmetric origin-destination tables, and different compositions of the bi-modal traffic (pairs of $n_{c}$ and $n_{b}$ ). The demand profile for cars is a typical peak-hour demand with a trapezoidal shape. For buses, the demand is determined by the number of lines and their operational frequency using data from SFMTA. Higher demand scenarios are also analyzed to generate various mode compositions. For the simulation experiments, the test network is modeled via the AIMSUN microscopic simulator. The simulation horizon for each experiment is $5.5 \mathrm{~h}$ and pairs of data $\left(n_{c}, n_{b}\right)$ are gathered every 5 min from the simulator. For each 5-min interval, $Q_{b}, Q_{c}$ are estimated as described above. Note that more than 20 scenarios with different dynamic demand characteristics are carried out to generate the corresponding traffic performance $Q$ for a wide range of $n_{c}$ and $n_{b}$. In the simulation, the dwell time was time-dependent with three different periods: the onset of the peak-hour, peak-hour and the offset of peak hour. For each time period, the average dwell time was fixed for all bus lines. However, the dwell times followed normal distribution, with a standard deviation around $20 \%$ of the mean. This means that in some cases of low demand, the buses could skip one stop. Some scenarios are analyzed later in more details.

Fig. 1(a) illustrates the 3D-vMFD for bi-modal traffic, $Q\left(n_{c}, n_{b}\right)$. As a first remark, Fig. 1(a) confirms the existence of a 3D- $v$ MFD for bi-modal networks, with a shape that depends on the accumulation of both cars and buses. To enable a better understanding of this figure, Fig. 1(b) displays the contour plot of the 3D-MFD on the $\left(n_{c}, n_{b}\right)$ plane, using the Delaunay triangulation/interpolation algorithm (De Berg et al., 2008) to estimate flow in a continuous space of the accumulation plane. The triangle in this figure indicates $\left(n_{c}, n_{b}\right)$ pairs, where the network operates close to the maximum throughput of the $3 \mathrm{D}-v$ MFD (values within the $80 \%$ range of the maximum flow). In particular, it captures the "optimal operational regime" of bi-modal traffic. City managers and practitioners could seek to derive management strategies to operate at this optimal 

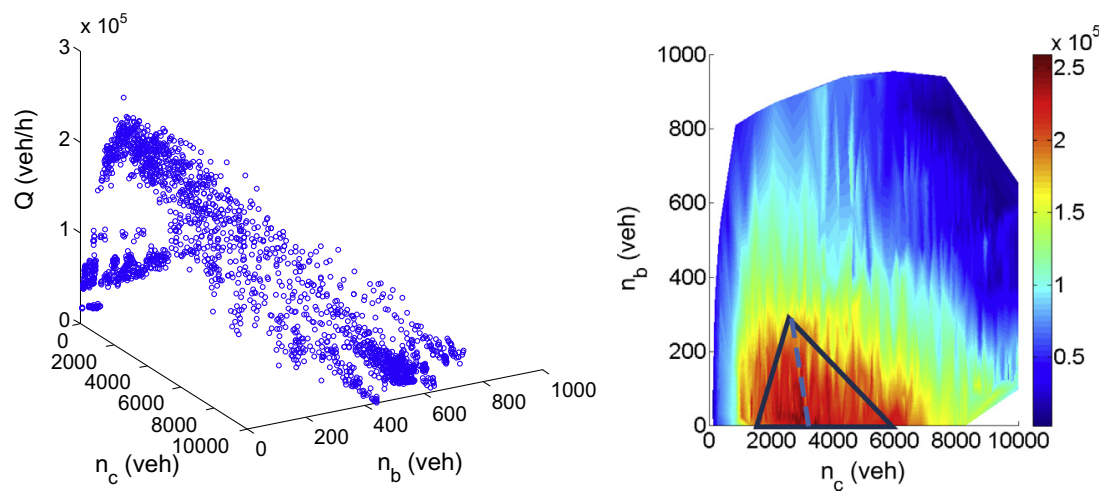

Fig. 1. (a) (left) The 3D-vMFD points for bi-modal traffic; and (b) (right) contour plot of the 3D-vMFD after interpolation by Delaunay triangulation. The color represents the value of circulating flow $Q$ (vehs/h).

regime. It can be seen that the flow $Q$ decreases monotonically as $n_{c}$ and $n_{b}$ increases, albeit with different slopes. Remarkably, the slope of buses is higher that the slope of cars. This indicates that effect of an additional bus in the network is much different than an additional car. A simple explanation is that buses make additional to traffic signal stops for passengers, and negatively influence traffic by creating stop-and-go phenomena. The figure also depicts critical accumulations of cars $\tilde{n}_{c}$ (rising line in the triangle) where $Q$ reaches its maximum for different values of $n_{b}$. The slope of the rising dashed line reflects that the capacity to serve cars has to be compromised in order to serve more buses, approximately $\tilde{n}_{c} \cong 3500-0.5 n_{b}$. As a general remark, the 3D-vMFD can be used by policy makers to exploit the trade-off between the operation of buses and cars and design more sustainable cities. Note also that the maximum value of the network flow occurs for $n_{b}=0$ and $n_{c}=3500$ because of the effect of bus stops. If one ignores the higher passenger bus occupancy, an optimal strategy to maximize vehicle flow is to operate at the $(3500,0)$ pair. The shape of the 3D-vMFD and the "optimal operational regime" depends in the geometry of the network, the signal settings and the public transport characteristics (location of stops, size and location of births, existence or not of transit signal priority etc.). As we show in the next section, a consideration of different vehicle occupancies for buses and cars in the estimation of network passenger flow will produce a different result.

\subsection{A three-dimensional MFD for passenger flows (3D-pMFD)}

Given the 3D-vMFD in Fig. 1, incoming flow can be controlled at the boundary of the network in order to operate at its "optimal operational regime". Existing methodologies for car-only perimeter control can be found in Keyvan-Ekbatani et al. (2012), Haddad et al. (2013), Haddad and Geroliminis (2012), Aboudolas and Geroliminis (2013) and elsewhere. However, these types of strategies are not able to consider that buses are more efficient modes due to higher passenger occupancy and they might restrict bus flow from entering the network. Before developing traffic management strategies for bi-modal systems with preferential treatment of buses, dynamics of passenger flows have to be investigated.

Denote $P$ the passenger flow in the bi-modal network, with $n_{c}$ and $n_{b}$. We are interested in observing $P=P\left(n_{c}, n_{b}\right)$. Denote $h_{m}$ the average number of on-board passenger occupancy per vehicle, $m \epsilon\{b, c\}$. We assume that car occupancy $h_{c}$ is constant $h_{c}=1.3$, while we estimate bus occupancy $h_{b}$ as an increasing function of the dwell times (similar to Zheng and Geroliminis, 2013). Dwell times are time-dependent, with three periods: on-set, during and off-set of peak hour. Dwell times are stochastic following a bounded normal distribution, so to capture some uncertainty in passenger demand (in the simulation, only dwell times are available). Then, by definition, total passenger flow $P$ can be expressed as

$$
P\left(n_{c}, n_{b}\right)=h_{c} Q_{c}\left(n_{c}, n_{b}\right)+h_{b} Q_{b}\left(n_{c}, n_{b}\right)
$$

We estimate passenger flows via the available simulated data, with the measurements of car flows $Q_{c}$, bus flows $Q_{b}$ and the estimated bus occupancy $h_{b}$ (direct simulation of passenger movements is not possible in the simulation). We use the same simulated flow data as the ones used to construct Fig. 1(a). Fig. 2(a) illustrates the 3D-pMFD relating accumulation of cars and buses to passenger flows. This figure presents a 3D-MFD like-shape for passenger flow, which looks similar with Fig. 1(a), but with higher maximum value, due to the $h_{b}$ term in Eq. (2). Nevertheless, a more careful look through the contour plot reveals significant differences.

Fig. 2(b) depicts the resulting contour plot of $P$ on the $\left(n_{c}, n_{b}\right)$ plane after applying the same triangulation algorithm as in Fig. 1(b). The polygon in this plot captures the "optimal operational regime" of passenger flow of bi-modal traffic. It can be seen that the shape of the region is significantly different from the one observed in Fig. 1(b). More precisely, for a given $n_{c}$ passenger flow $P$ first monotonically increases as $n_{b}$ increases to a critical $\tilde{n}_{b}$ and then decreases for $n_{b}>\tilde{n}_{b}$. Thus, having buses in the network will significantly increase the efficiency of the system but overloading the network with buses will 

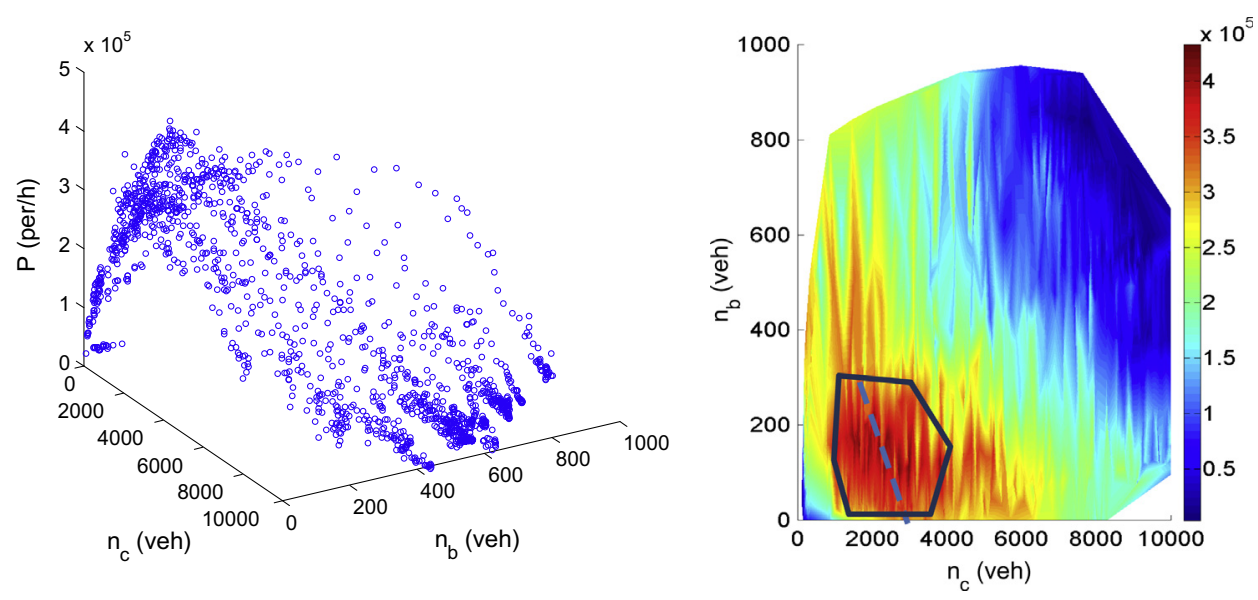

Fig. 2. (a) (left) The 3D-pMFD for bi-modal passenger traffic; and (b) (right) contour plot of the 3D-pMFD, the color represents the value of flow $P$ (pers/h).

eventually cause delays for all vehicles and reduce passenger throughput. The figure also displays that $\tilde{n}_{c}$ in this case (rising dotted line in the polygon) has a clear tendency of becoming smaller as $n_{b}$ increases, $\tilde{n}_{c} \cong 3500-6 n_{b}$. The slope of the rising line reflects that only a slight increase of buses can allow a large reduction of cars to maintain the same passenger flow, which was not the case in the $Q\left(n_{c}, n_{b}\right)$ plot. The maximum network flow occurs now for a positive $n_{b}$, the pair (2800, 120). It can be foreseen that more buses can be deployed in the network to succeed a higher passenger flow if dedicated bus lanes are provided. For example, Zheng and Geroliminis (2013) tried to optimize passenger flows in a bi-modal network by redistributing the road infrastructure and offering a fraction of roads for dedicated bus lanes.

\subsection{A functional form of $Q\left(n_{c}, n_{b}\right)$ and $P\left(n_{c}, n_{b}\right)$}

Given that evaluating $Q\left(n_{c}, n_{b}\right)$ in (1) for many pairs of $n_{c}$ and $n_{b}$ is tedious, we propose instead using an analytical function that approximates the 3D-vMFD in Fig. 1(a). Given the properties of $Q\left(n_{c}, n_{b}\right)$ described in the previous section, we consider an exponential-family function, which has useful algebraic properties. In general exponential families are in a sense very natural distributions to consider with plenty of applications in physics and statistics. Note that parabolic families do not provide a good fit given their single value of the 2 nd derivative. To this end, we consider the following exponential flow function for data fitting:

$$
Q\left(n_{c}, n_{b}\right)=a\left(n_{c}+n_{b}\right) e^{b n_{c}^{2}+c n_{b}^{2}+d n_{c} n_{b}+e n_{c}+f n_{b}}
$$

where $a, b, c, d, e, f$ are model parameters. The parameter values should be specified so as to minimize the deviation of model (1) from the corresponding measured values $Q^{\prime}$. This function can be considered as a generalization of the Drake's exponential function for a single-mode fundamental diagram between flow and density. An unconstrained estimation will not be consistent with the physics of traffic, thus constraints ( $4 \mathrm{~b}$ ) are added. To this end, we estimate the values by Least-Squares parameter estimation for the given simulated data $Q^{\prime}$ in Fig. 1(b). The constrained parameter estimation problem is formulated as follows (P1):

$$
\min _{a, b, c, d, e . f} Z=\left\|\boldsymbol{Q}-\boldsymbol{Q}^{\prime}\right\|^{2}
$$

subject to

$$
\left\{\begin{array}{crr}
Q \geqslant 0, & \text { for } & 0 \leqslant n_{c} \leqslant \max \left(n_{c}\right) \text { and } 0 \leqslant n_{b} \leqslant \max \left(n_{b}\right), \\
\frac{\partial V\left(n_{c}, n_{b}\right)}{n_{c}} \leqslant 0, & \text { for } 0 \leqslant n_{b} \leqslant \max \left(n_{b}\right), \\
\frac{\partial V\left(n_{c}, n_{b}\right)}{\partial n_{b}} \leqslant 0, & \text { for } 0 \leqslant n_{c} \leqslant \max \left(n_{c}\right),
\end{array}\right.
$$

where $V$ is the space-mean speed in the network, $\mathbf{Q} \mathbf{Q}^{\prime}$ are vectors with elements of the model (2) and the simulated data for each 5 min sample interval, respectively. The space-mean speed is given by $V\left(n_{c}, n_{b}\right) \stackrel{\text { def }}{=} Q L /\left(n_{c}+n_{b}\right)$, where $L$ is the average link length of the network. The first constraint in (4b) guarantees non-negative flows for values of accumulation between zero and jammed. The second and the third constraint emphasize that the space-mean speed of all vehicles should decrease monotonically as $n_{c}$ and $n_{b}$ increase.

The constrained parameter estimation problem (P1) is nonlinear and is solved through the Sequential Quadratic Programming (SQP) approach. SQP method solves a sequence of optimization sub-problems, each of which optimizes a quadratic model of the objective subject to a linear relaxation of the constraints. We apply this algorithm for multiple initial values 
(around 1000) to avoid convergence to local minima, which might be the case of non-smooth data. The estimated parameters resulting from the optimization problem are: $\{a, b, c, d, e, f\}=\left\{1.95 \cdot 10^{2},-2.34 \cdot 10^{-9}, 5.28 \cdot 10^{-7}, 6.34 \cdot 10^{-8},-2.92 \cdot 10^{-4}\right.$, $-1.50 \cdot 10^{-3}$ \}. The $T$-test result indicates that all six parameters are statistically significant ( $p$-value almost zero). Fig. 3(a) illustrates the results of fitting model (P1) with the estimated parameters to the simulated data. An $R$-square value of 0.91 (close to 1) indicates that the resulting 3D- $v$ MFD fits well with the data and all physical constraints (4b) are satisfied. We also verify the adequate fitting performance of the model by 3 different demand scenarios, which were not used for model estimation and have different demand profiles. Fig. 3(b) depicts the contour plot of the 3D surface on the $\left(n_{c}, n_{b}\right)$ plane. Comparing Fig. 3(b) with Fig. 1(b), we can see that most patterns observed closely matches each other except the area for very high values of buses $n_{b}>600$ due to lack of simulated data. Nevertheless, these states cannot be easily observed in real systems. Moreover, it can be seen that the "optimal operational regime" (triangle in Fig. 1(b)) of bi-modal traffic is reproduced in a very similar way.

We perform a similar optimization for fitting the measured passenger flow $P$ with parameters $a^{\prime}, b^{\prime}, c^{\prime}, d^{\prime}, e^{\prime}, f^{\prime}, g^{\prime}$ for an exponential function $P\left(n_{c}, n_{b}\right)=a^{\prime}\left(n_{c}+g^{\prime} n_{b}\right) e^{b^{\prime} n_{c}^{2}+c^{\prime} n_{b}^{2}+d^{\prime} n_{c} n_{b}+e^{\prime} n_{c}+f^{\prime} n_{b}}$. Denote this parameter estimation problem as (P2). Solving (P2), we get $\left\{a^{\prime}, b^{\prime}, c^{\prime}, d^{\prime}, e^{\prime}, f^{\prime}, g^{\prime}\right\}=\left\{3.46 \cdot 10^{2}, 6.41 \cdot 10^{-10},-2.27 \cdot 10^{-6},-1.14 \cdot 10^{-7},-3.77 \cdot 10^{-4},-5.30 \cdot 10^{-4}, 3.66 \cdot 10^{0}\right\}$. Fig 3(c) depicts the result of the fitting model with the parameters. An R-test value of 0.91 (close to 1 ) indicates a decent model fitting, as well. Fig. 3(b) depicts the contour plot of the 3D surface on the $\left(n_{c}, n_{b}\right)$ plane. Nevertheless, by comparing the parameters of the two models, no physical interpretations can be made. In Section 3 we provide an analytical derivation to estimate $P\left(n_{c}, n_{b}\right)$ from $Q\left(n_{c}, n_{b}\right)$, without the need of curve-fitting, which unveil interesting properties of network traffic flows.

\subsection{Bus-Car Unit (BCU) equivalent}

In transportation planning and analysis of bi-modal systems, it is a common approach to assume that each bus is equivalent to a constant number of cars independent of the level of congestion and the densities of each mode. This simplifies the derivations of different performance measures. Nevertheless, it is clear from the explanation of the previous section that the influence of buses in the network performance depends on the state of the system $\left(n_{c}, n_{b}\right)$ and cannot be considered constant. Thus, a static BCU consideration is insufficient to reflect the dynamic relationship between bus/car accumulation and congestion. For this reason, we propose here an analytical estimation of the BCU value as a function of the accumulation of both modes.

Let us consider that the average network speed is $V\left(n_{c}, n_{b}\right) \stackrel{\text { def }}{=} Q\left(n_{c}, n_{b}\right) L /\left(n_{b}+n_{c}\right)$. We assume that for a given $n_{b}, V\left(n_{c}, n_{b}\right)$ is monotonically decreasing function of $n_{c}$ and the same assumption for $n_{c}$ (see the 2 nd and 3rd constraint in Eq. (4b)). Given that $V\left(n_{c}, n_{b}\right)$ is a continuous and differentiable function, we can estimate the effect that buses and cars have in speed $V$ for a
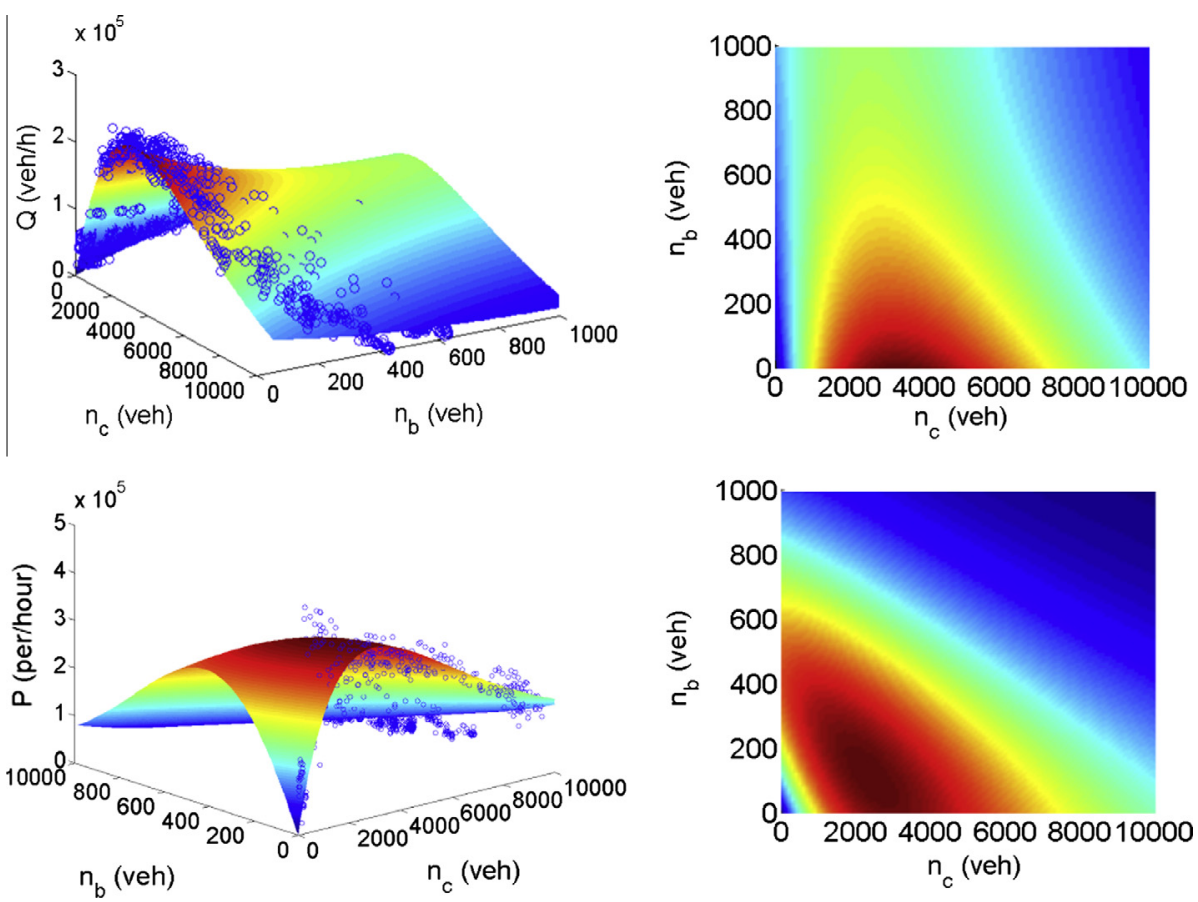

Fig. 3. (a) (top left) The exponential $Q\left(n_{c}, n_{b}\right)$; (b) (top right) contour plot of $Q\left(n_{c}, n_{b}\right)$; (c) (down left) the exponential $P\left(n_{c}, n_{b}\right)$ and (d) (down right) contour plot of $P\left(n_{c}, n_{b}\right)$. 
given $\left(n_{c}, n_{b}\right)$ pair. Mathematically speaking, $B C U$ is defined and estimated as the ratio of partial derivatives with respect to $n_{c}$ and $n_{b}$

$$
\operatorname{BCU}\left(n_{c}, n_{b}\right)=\frac{\frac{\partial V\left(n_{c}, n_{b}\right)}{\partial n_{b}}}{\frac{\partial V\left(n_{c}, n_{b}\right)}{\partial n_{c}}}
$$

By assuming the closed form solution of $Q\left(n_{c}, n_{b}\right)$ in (3) and after some algebra we obtain

$$
B C U\left(n_{c}, n_{b}\right)=\frac{2 c n_{b}+d n_{c}+f}{d n_{b}+2 b n_{c}+e}
$$

Fig. 4 plots $B C U\left(n_{c}, n_{b}\right)$ which is a monotonically decreasing function of vehicle accumulations. Physically speaking, Fig. 4 highlights that the effect of buses in the network speed is smaller as the network is more congested. This is because dwell times at bus stops are a smaller fraction of car travel time compared to free-flow conditions. For example, in case of $10 \%$ bus accumulation, $B C U$ values range between 5.0 for free-flow conditions to 3.5 for highly congested conditions.Nevertheless, Eq. (5a) might not be straightforward to estimate with real data without curve fitting, due to the scatter of the MFD measurements, that might make the estimation of derivatives complicated. An approximation of (5a) based on engineering principles, $B C U^{*}\left(n_{c}, n_{b}\right)$, can be obtained if we consider for a given pair of $\left(n_{c}, n_{b}\right)$, an equivalent system with "cars only" that satisfies $V\left(n_{c 0}, 0\right)=V\left(n_{c}, n_{b}\right)$, where $n_{c 0}$ is the accumulation of cars for a car-only network, i.e. $n_{b}=0$. Thus, $B C U^{*}\left(n_{c}, n_{b}\right)$ is the solution of the equation $n_{c 0}=n_{c}+B C U^{*} \cdot n_{b}$. In other words, $B C U$ expresses an equivalent system with cars only that has the same space-mean speed with the mixed-traffic network. Then the average speed can be rewritten as

$$
V\left(n_{c}+B C U^{*} \cdot n_{b}, 0\right)=V\left(n_{c}, n_{b}\right)
$$

Combining the functional form (3) and Eq. (6a), we obtain an analytical solution of $B C U$ as a solution of the quadratic equation $b n_{b} B C U^{* 2}+\left(2 b n_{c}+e\right) B C U^{*}-c n_{b}-d n_{c}-f=0$, which is given by

$$
B C U^{*}\left(n_{c}, n_{b}\right)=\frac{-\left(2 b n_{c}+e\right)-\sqrt{\left(2 b n_{c}+e\right)^{2}+4 b n_{b}\left(c n_{b}+d n_{c}+f\right)}}{2 b n_{b}}
$$

Given that the value of $b$ is relatively small, $B C U$ estimation can be approximated (error less than 0.01 by the solution of $e B C U-c n_{b}-d n_{c}-f=0$ and therefore gives the $B C U^{*}$ a plane shape). These results also indicate that the $B C U^{*}$ value for buses becomes smaller as congestion increases. While this result might be considered as counter-intuitive, it is consistent with the physics of traffic because the effect of bus related stops is a smaller part of the total travel time for congested conditions.

\subsection{A macroscopic fundamental diagram for buses operating in dedicated lanes}

As dedicated bus lane allocation is a sustainable strategy for multimodal urban transportation systems with preferential treatment of higher occupancy modes of transport, it deserves the effort to look into a city-wide MFD for buses running in dedicated bus lanes. While a detailed analysis is beyond the scope of this paper, we perform an analysis where all the bus lines perform in dedicated lanes. To this end, the existing test site is updated and one lane in each road of the city center with public transport lines is assigned to buses if space is available. The resulted bus-lane network is well connected and includes about $5 \mathrm{~km}$ of dedicated bus lanes. To derive and investigate the shape of the MFD for buses, simulations are performed with a field-applied fixed-time plan and a fixed number of bus lines. To account for demand effects of the bi-modal traffic composition, ten runs were carried out for a 4-h time-dependent scenario with different car demand and bus frequency. Additionally, two scenarios (with five runs each) based on different dwell time of buses were defined in order to investigate the impact of the dwell time in the shape of the MFD for buses. Fig. 5 displays the MFD for buses resulting for the aforementioned runs. This figure plots the network flow-density relationship (buses $/ 5 \mathrm{~min}$ vs. buses/km) in the network for the whole simulation time period. Each measurement point in the diagram corresponds to 5 min interval. Fig. 5(a) confirms the

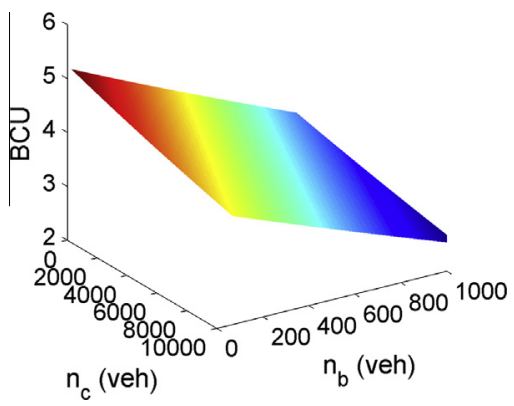

Fig. 4. A $3 D$ diagram relating accumulation of cars and buses with BCU. 

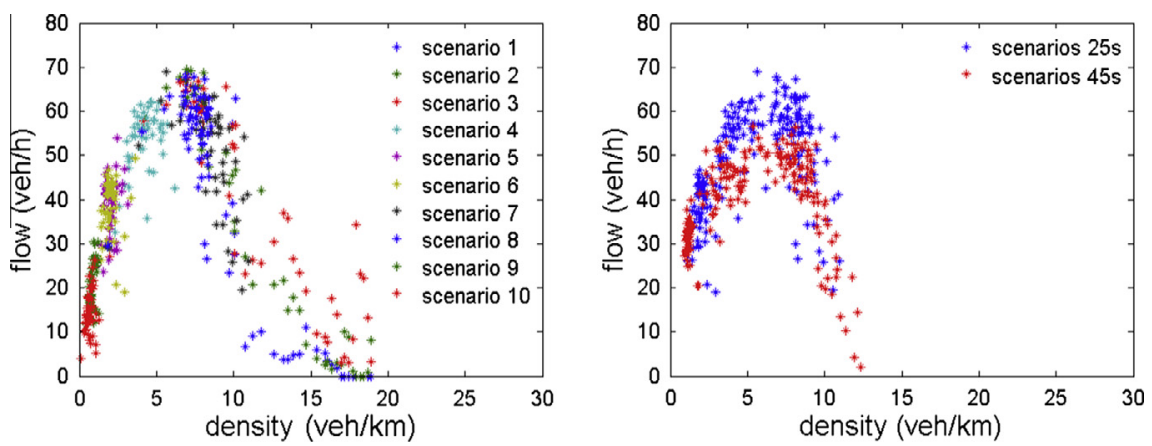

Fig. 5. (a) (left) The MFD for buses for ten runs with different bi-modal traffic patterns; and (b) (right) the impact of different average dwell times of buses on the MFD.

existence of an MFD for buses with moderate scatter across different scenarios. Note that the value of critical density and maximum flow are considerably smaller compared to the ones of the vehicle MFD (critical density around 7 buses/km and capacity around 70 buses/h).

It can be seen that the free flow speed in the bus lanes is around $25 \mathrm{~km} / \mathrm{h}$ and the maximum flow occurs in a density range from 5 to 10 buses per $\mathrm{km}$. If the density is allowed to increase, then the dedicated bus lanes become severely congested. The flow decreases with density (negative slope) and the network can lead to gridlock. This MFD like-shape for buses operating in dedicated lanes is quite conforming to the MFD for cars and mixed traffic. An interesting observation is that the diagram indicates a high flow scatter for the critical bus density $\tilde{k}_{b}$. This difference is attributed to different traffic patterns for the cars and the interaction of the bi-modal traffic at the intersections that could lead to partially block of the dedicated bus lanes due to spillbacks. Besides bus frequency, the effect of the dwell times in the shape of the MFD is analyzed as longer dwell times reduce the average speed and flow. Fig. 5(b) illustrates the impact of different dwell time of buses on the MFD, plotting the MFD for buses resulting for multiple scenarios, where half scenarios have average dwell time of $25 \mathrm{~s}$ and the other $45 \mathrm{~s}$. It can be seen that the higher dwell times lead to smaller flow capacity (in buses/h), by about $15 \%$. But, if we consider that bus occupancy might be almost double with higher dwell times, then the efficiency of the specific dedicated bus lanes in terms of passengers significantly increases. A higher number of bays to simultaneously serve buses at the same stop might also increase the network bus capacity. Optimal redistribution of road space between buses and cars, and the effect of such strategy in the mode choice are not described in this work. A theoretical approach towards this direction has been introduced in Zheng and Geroliminis (2013).

\section{An analytical model for the estimation of passenger flows}

To maximize the passenger flow of a network and thus realize the efficiency of a multimodal network, we should seek a 3D-pMFD that can capture the dynamics of passenger flows but also can be derived from (3) rather than curve fitting. To this end, we describe a derivation of the analytical form of the passenger flow 3D-pMFD based on the vehicle flow 3D- $v$ MFD in this section. We further show that by partitioning the network into regions with homogeneous traffic and mode composition, the analytical model is able to reproduce the passenger flow pattern as observed from the data.

\subsection{The derivation of a bi-modal MFD for passenger flow}

Let us investigate the analytical derivation of $P$ given the analytical form of $Q$ in (3). Our first objective is to estimate flows $Q_{c}$ and $Q_{b}$ for each mode by utilizing $Q, n_{c}$ and $n_{b}$. To this end, we first consider that $V=\left(v_{c} n_{c}+v_{b} n_{b}\right) /\left(n_{c}+n_{b}\right)$ where $v_{c}$ is the speed of car and $v_{b}$ the speed of bus, and $Q$ is given by

$$
Q=Q_{c}+Q_{b}=\left(v_{c} n_{c}+v_{b} n_{b}\right) / L
$$

To estimate the individual flows of each mode, a relationship between their speeds is necessary, $v_{b}=v_{c}\left(n_{c}, n_{b}\right)$. To obtain $v_{b}$ as function of $v_{c}$, we utilize a first order approximation, a linear model between $v_{c}$ and $v_{b}$

$$
v_{b} \cong \theta v_{c}+\beta
$$

where $\theta$ and $\beta$ are parameters that can be estimated with real data and depend on the operational characteristics of the buses, the network topology and the composition of traffic in the network. Alternatively, one can use a more detailed speed model, as for example the one proposed in Zheng and Geroliminis (2013), where the average distance between successive bus stops and the average dwell time at a bus stop influence the relation between $v_{b}$ and $v_{c}$. We show later that model (8) can give a decent approximation of $v_{b}$. Nevertheless, parameters $\theta$ and $\beta$ might not be universal and depend on the ratio of buses and cars in the network, $n_{c} / n_{b}$. The aforementioned models might be less accurate in case of bus bunching, where multiple buses are queued in a bus stop with limited berths for boarding and alighting. A more detailed queuing model can 
be derived in such a case. By introducing (8) in (7), we can obtain the analytical form of $v_{c}$. Then after some algebra we estimate $Q_{c}$ and $Q_{b}$ as follows $\left(Q\left(n_{c}, n_{b}\right)\right.$ is noted as $\left.Q\right)$ :

$$
\begin{aligned}
& Q_{c}=v_{c} n_{c}=\frac{Q L-\beta n_{b}}{\left(n_{c}+\theta n_{b}\right)} \frac{n_{c}}{L} \\
& Q_{b}=v_{b} n_{b}=\left(\frac{Q L-\beta n_{b}}{\left(n_{c}+\theta n_{b}\right)} \theta+\beta\right) \frac{n_{b}}{L}
\end{aligned}
$$

A physical interpretation of such equations is that as bus is a slower mode, the fraction of bus flow is smaller than the fraction of bus accumulations. Then we derive $P\left(n_{c}, n_{b}\right)$ by combining (2), (9), and (10).

$$
P\left(n_{c}, n_{b}\right)=h_{c}\left(\frac{Q\left(n_{c}, n_{b}\right) L-\beta n_{b}}{\left(n_{c}+\theta n_{b}\right)}\right) \frac{n_{c}}{L}+h_{b}\left(\frac{Q\left(n_{c}, n_{b}\right) L-\beta n_{b}}{\left(n_{c}+\theta n_{b}\right)} \theta+\beta\right) \frac{n_{b}}{L}
$$

Note that the 3D-pMFD is a function of the 3D- $v$ MFD, $Q\left(n_{c}, n_{b}\right)$, the individual accumulations of buses and cars, $\left(n_{c}, n_{b}\right)$ and the average link length, $L$. A validation of the analytical model is provided later.

\subsection{Network partitioning}

By using a single value of $\theta$ and $\beta$ in (8) for the whole network, one assumes that the interaction between cars and buses is homogeneous in space and time. Nevertheless, the analytical estimation in (11) cannot accurately reproduce $P\left(n_{c}, n_{b}\right)$, if $Q\left(n_{c}, n_{b}\right)$ is assumed to follow the exponential model (3). The reason is simple, bus lines are not evenly distributed in the studied network. The ratio $\delta_{i}$ between the density of bus $k_{b i}$ and density of cars $k_{c i}$ (where $i$ is a link) is much higher in the center of the network, where there is higher frequency and density of bus lines. Fig. 6(a) displays the bus routes of the simulated network, which match the real ones. Note that some lines share links with the others in the center part of the network and that not all the lines can be visualized in the figure. We can observe that, the center area of the network $\left(x \in[2500,4000], y \epsilon\right.$ [3500 4500]) includes more bus lines than the other parts of the network. Let us take $\delta_{i}$ at a certain time interval during the simulation, when the network is operating at its maximum flow rate. Fig. 6(b) illustrates the scale distribution of $\delta_{i}$ by a gray-scale plot, where a darker color indicates a higher value of $\delta_{i}$. This result shows that there exists a strong heterogeneity in the spatial distribution of $\delta_{i}$, especially when comparing the mode composition between the center area (roughly identified) and the rest of the network. Note also that the same part of the network with higher $\delta_{i}$, experiences more congestion than the remaining part.

To this end, we partition this network for the sake of homogeneity of $\delta_{i}$ within each partitioned regions. This partitioning builds in a clustering algorithm proposed in Ji and Geroliminis (2012) for heterogeneously congested networks. The objectives of partitioning are to obtain (i) small variance of link ratio $\delta_{i}$ within a cluster and (ii) spatial compactness of each cluster, which makes feasible the application of perimeter control strategies. The partitioning mechanism consists of three consecutive algorithms. Firstly, an over segmenting of the network is provided by a sophisticated algorithm (Shi and Malik, 2000). Secondly, a merging algorithm is developed based on initial segmenting and a rough partitioning of the network is obtained. Finally, a boundary adjustment algorithm is designed to further improve the quality of partitioning by decreasing the variance of $\delta_{i}$ while keeping the spatial compactness of the clusters. The similarity between links $i$ and $j, w(i, j)$, is given by
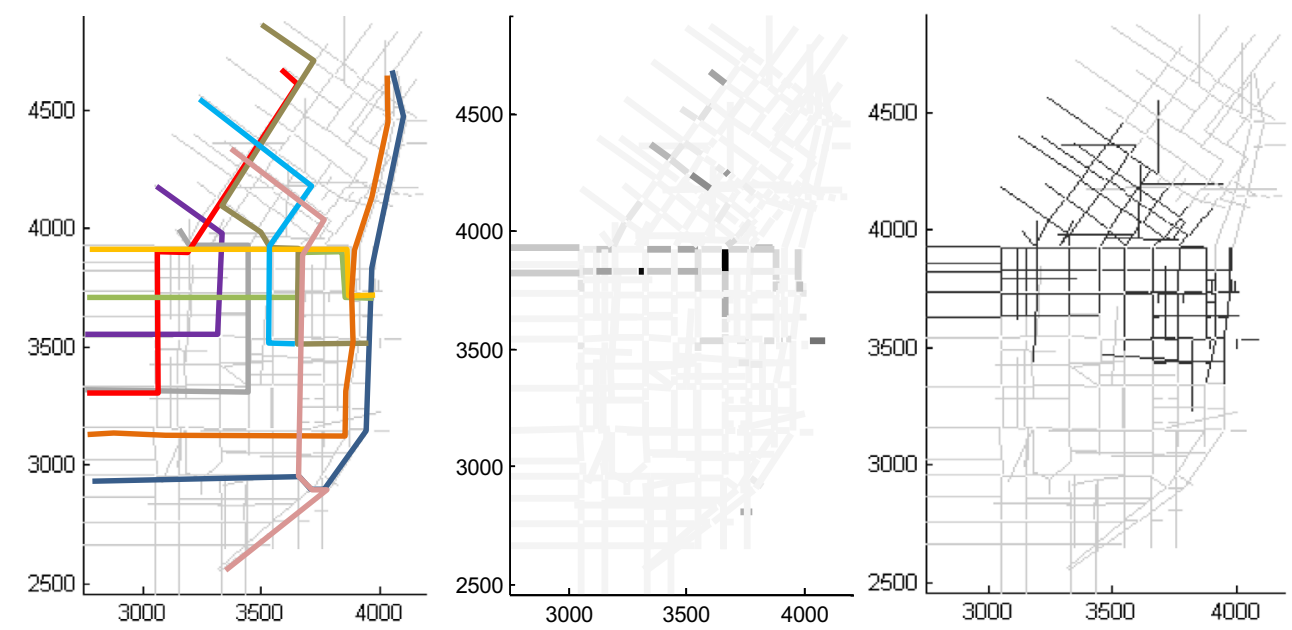

Fig. 6. Illustration of the partitioned network: (a) (left) Layout of the bus lines over the network; (b) (middle) contour plot of link ratio $\delta_{i}$; and (c) (right) result of partitioning. 


$$
w(i, j)=\left\{\begin{array}{cc}
\exp \left(-\left(\delta_{i}-\delta_{j}\right)^{2}\right), & \text { if } \quad r(i, j)=1 \\
0, & 0 . w .
\end{array}\right.
$$

where $r(i, j)$ is the spatial distance between two links denoted by the length of the shortest part between $i$ and $j$. Eq. (12) simply explains that if two links are not adjacent, their similarity is negligible. Such an approach, can guarantee that the developed clusters will be well-connected (compact) and the topological characteristics influence links in the same cluster. For a detailed description, the reader should refer to Ji and Geroliminis (2012). The studied network is divided into two regions (clusters) as shown in Fig. 6(c) after the application of the algorithm. Let us denote these two regions the "center region" and the "outside region" respectively, where the center region has more bus lines. We can see that this result closely matches what we have observed from the data in Fig. 6(b). Note that, the partition algorithm by its nature tries to give a compact shape with smooth boundaries of the partitioned regions. This explains why the exact shape of the area with high value of $\delta_{i}$ is not fully obtained. The objective of partitioning is satisfied as the mean value of $\delta_{i}$ in the center region is much larger than the one in the outside region, $\left(\delta_{\text {center }}, \delta_{\text {outside }}\right)=(0.40,0.04)$. The mean value of $\delta_{i}$ in the whole network is 0.20 (around 16\% of vehicles are buses). The standard deviation of $\delta_{i}$ is 0.45 for the center region and 0.12 for the outside region, which is smaller compared to 0.76 for the whole network. This means that the algorithm succeeds to cluster together links with more similar $\delta_{i}$. Note that standard deviations are high because in both sub-regions there are plenty of links with no buses, $\delta_{i}=0$ and in the center region there are some links with bus bunching (and high $\delta_{i}$ ).

We are interested in investigating if this partition reduces the heterogeneity of congestion as well. Therefore, we calculate the total density $k_{i}\left(k_{i}=k_{c i}+k_{b i}\right)$ of link $i$ in each partitioned region and comparing the variance of $k_{i}$ within each region, when the network vehicle flow is maximum. The mean values of $k_{i}$ are $15 \mathrm{veh} / \mathrm{km}$ for the whole network, while $18 \mathrm{veh} / \mathrm{km}$ for the center region and $13 \mathrm{veh} / \mathrm{km}$ for the outside region. The standard deviation of $k_{i}$ is $9 \mathrm{veh} / \mathrm{km}$ for the center region and $6 \mathrm{veh} /$ $\mathrm{km}$ for the outside region, which is again smaller compared to a value of $16 \mathrm{veh} / \mathrm{km}$ for the whole network. It shows that a mode-composition-based partitioning is sufficient to reduce heterogeneity of 3D-MFD. In reality this may not always be the case, if the scale of flow densities is not similar among links. In those cases, one may apply a partition algorithm based on both $\delta_{i}$ and $k_{i}$, e.g. using a different similarity function in (12). As we show in the next section, the resulting partitioning is sufficient for understanding the traffic heterogeneity and provides a good approximation of the 3D- $p$ MFD.

Furthermore, we verify the usefulness of the partitioning from a traffic point of view, by examining the time when each region reaches congestion. Here, "congestion" starts when network flow drops after the network reaches its maximum flow. To compare regions of different sizes, instead of accumulations we utilize densities. Fig. 7(a) shows the MFD (vehicle flow $Q=Q_{b}+Q_{c}$ vs. vehicle density $k=\sum_{i} k_{i}$ ) of the center region, the outside region and the whole network respectively, for one scenario. Density of buses and cars $\left(k_{b}, k_{c}\right)$ are estimated by dividing accumulations with the total lane kilometers utilized by each mode, $L_{b}^{\text {center }}=24 \ln -\mathrm{km}, L_{c}^{\text {center }}=37 \ln -\mathrm{km}, L_{b}^{\text {outside }}=26 \ln -\mathrm{km}, L_{c}^{\text {outside }}=64 \ln -\mathrm{km},\{b, c\}$ for bus and car respectively. The start of congestion in each region is indicated by an arrow with the corresponding timestamp attached. We can observe that the center region reaches congestion first, half hour earlier than the outside region. Fig. 7(b) displays the time series of the average density of the two regions respectively. Note also, that density in the center region is consistently higher than in the outer region between 9:30 and 12:00. Thus, a static partitioning (based on the conditions at 10am) succeeds to identify the more congested region of the network. This conclusion should not be universal as in case of different demand profiles and congestion propagation in time and space, partitioning should be re-evaluated in a dynamic way. The direction of dynamic partitioning is a challenging future area of research. The fact that regions of a network have various levels of congestion is important, as it indicates that different traffic management measures should be applied for the two regions based on their individual traffic states.

\subsection{Vehicle 3D-vMFD after partitioning}

Let us now show the 3D- $v$ MFD for the partitioned regions. We utilize the data of the same scenarios as in Fig. 1. To have the scale of the MFDs of the two regions comparable, we normalize the values of accumulations and reproduce densities. Denote density of cars $k_{c}$ and density of buses $k_{b}$, as estimated in the previous section. Given that the one region is larger
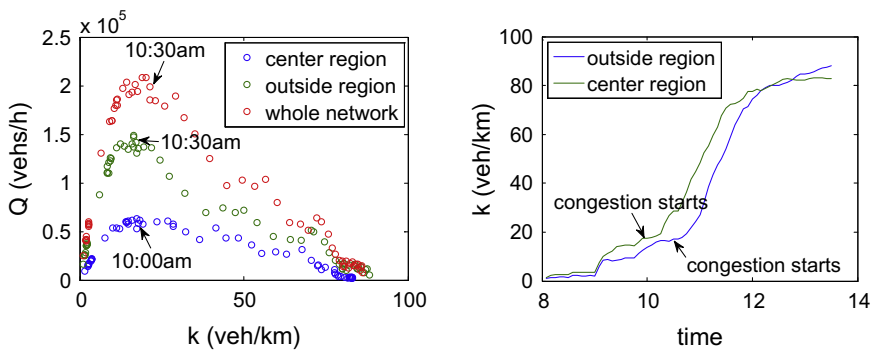

Fig. 7. Comparing the time each region reaches congestion in (a) (left) network flow-density relation and (b) (right) density time-series. 
than the other, we also normalize network flows by the maximum value of flow observed in each region. The normalized 3D$v$ MFDs for the two partitioned regions are plotted in Fig. 8(a). $k_{b}$ of the center region has a higher range of values, as the region is covered by more bus lines. Nevertheless, the general pattern observable in Fig. 8(b) and (c) is similar to the 3D$v$ MFD as observed in Fig. 3, especially the values of critical densities (for example 35vh/km $\times 100 \mathrm{ln}$-km $=3500 \mathrm{veh}$ which is the critical accumulation of Fig. 3b). Given the 3D- $v$ MFD of each region, specific management measures such as perimeter signal control can be applied with the objective of operating at the region's optimal regime.

\subsection{Passenger 3D-pMFD after partitioning}

Let us compare the resulting passenger dynamics of one scenario before and after partitioning. Both vehicle and the passenger MFDs are displayed in Fig. 9(a) for the whole network. Given that ratio $\delta$ is not significantly varying with time during the specific scenario, passenger flow $P(n)$ vs. total accumulation $n=n_{b}+n_{c}$ is displayed. Even without partitioning the network has a low scatter MFD with a well-defined maximum flow and a clear value of critical accumulation (Fig. 9(a)). The vehicular flow MFD, $Q(n)$, has a similar shape, but the value of critical accumulation is larger.

Let us have a more careful look in the results after partitioning. Fig. 9(b) plots $n_{b}$ vs. $n_{c}$ with time for the two partitioned regions. It can be seen that the center region has a $\delta$ of about 9\%, which is 6 times larger than $\delta$ in the outside region, where $\delta$ is the ratio $\sum_{i} n_{b i} / \sum_{i} n_{c i}, i$ is the link. Fig. 9(c) depicts the space-mean speed for each region and mode. The dashed lines indicate the fitting results of Eq. (8). Parameter $\theta$ for the center region is 0.3 and for the outside region is 0.5 , while $\beta$ is 0 in both cases. The resulting linear relationship is consistent with the proposed approximation in (8). If Eqs. (8)-(11) were applied to estimate $P(n)$ for the whole network, errors would not be negligible because of differences in $\delta$ and $\theta$ between the two regions. Fig. 9d shows the estimated $P\left(n_{c}, n_{b}\right)$ for the center and outside region after applying (8)-(10).

Let's now consider the performance of $(8)-(11)$ for the partitioned regions for three different scenarios with various bus demands, denoted as " $s_{1}$ ", " $s_{2}$ " and " $s_{3}$ ". After applying the partitioning algorithm, $\delta$ and $\theta$ are estimated for the center and outside region and passenger MFDs are plotted. Note that partitioning is static and is not re-estimated for different demand scenarios, because similar performance with respect to homogeneity metrics is achieved with Section 3.2. Nevertheless, dynamic partitioning algorithms are expected to improve the homogeneity of congestion distribution, especially for networks with propagation in both time and space and multiple pockets of congestion.

The total network passenger flow is estimated as

$$
P\left(n_{c}(t), n_{b}(t)\right)=P_{1}\left(n_{c 1}(t), n_{b 1}(t)\right)+P_{2}\left(n_{c 2}(t), n_{b 2}(t)\right)
$$

where indices 1 and 2 represent the two partitioned regions, center and outside. Fig. 10(a) shows accumulation of buses and cars for the entire network for the three scenarios. The red dotted line ("s $s_{3}$ ") represents the same scenario as of Fig. 9. The orange circles highlight the accumulation pairs where maximum flow is achieved in each scenario. Note the different values of mode composition $\delta$ for each scenario. Fig. 10(b) depicts the corresponding $P\left(n_{c}, n_{b}\right)$ of the three different scenarios, and the $\delta$ of each scenario is projected on the $\left(n_{c}, n_{b}\right)$ plane. We can see that, by applying the estimated $\theta$ based on partitioning, the analytical model is able to reproduce the important patterns as observed in Fig. 2(b): (i) $P$ increases and then decreases as $n_{b}$ increases, (ii) the critical accumulation largely shifts towards the left, and (iii) there exists an "optimal operational regime" where the same value of maximum $P$ (close to critical value) can be achieved with different combinations of $n_{b}$ and $n_{c}$.
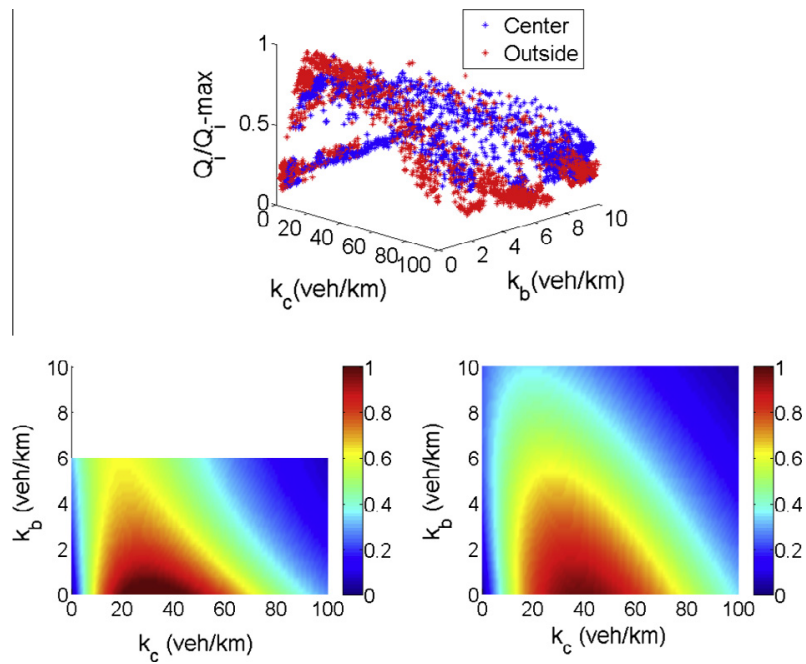

Fig. 8. (a) (top) The normalized 3D-MFD of the partitioned regions; the contour plots on ( $k_{c}, k_{b}$ ) plane for (b) (down left) the center region and (c) (down right) the outside region. 

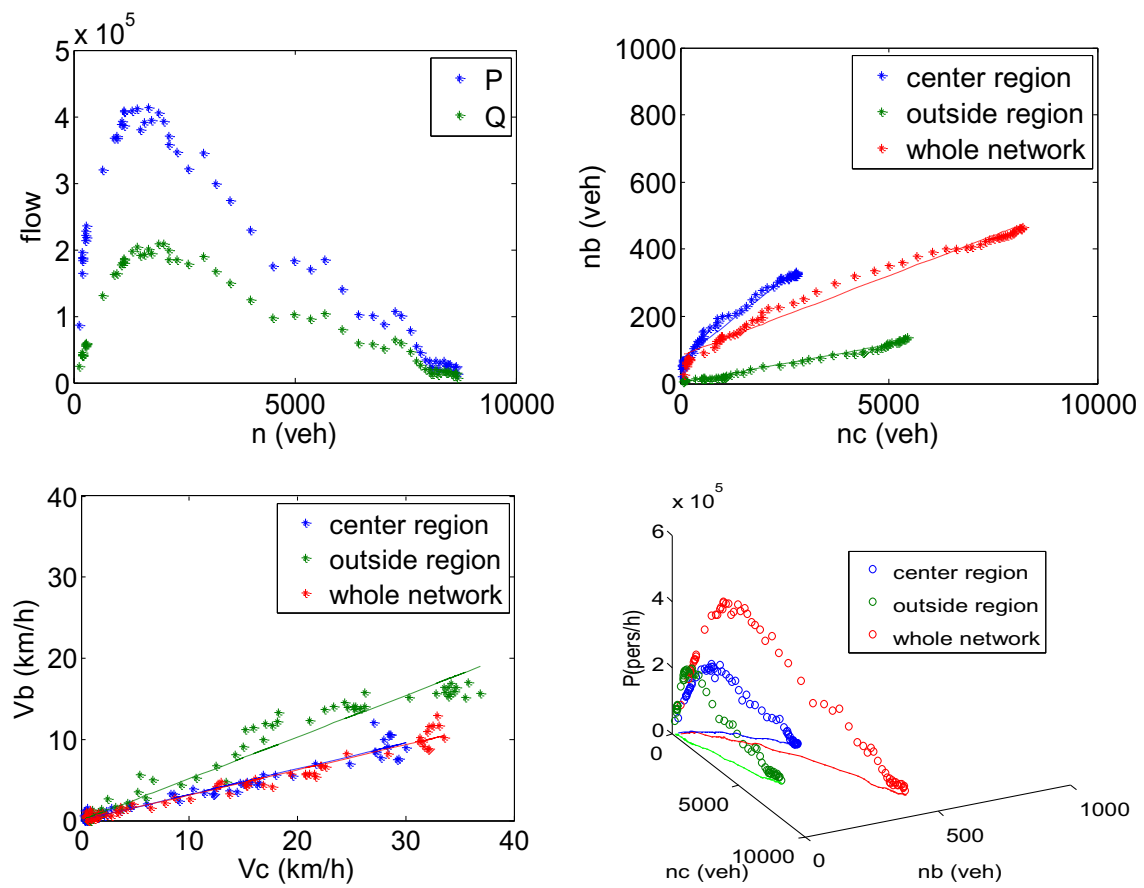

Fig. 9. (a) (top left) Passenger MFD from the simulation; (b) (top right) $n_{b}$ vs. $n_{c}$; (c) (down left) $v_{b}$ vs. $v_{c}$; and (d) (down right) the estimated passenger MFD for the two partitioned regions and the whole network.
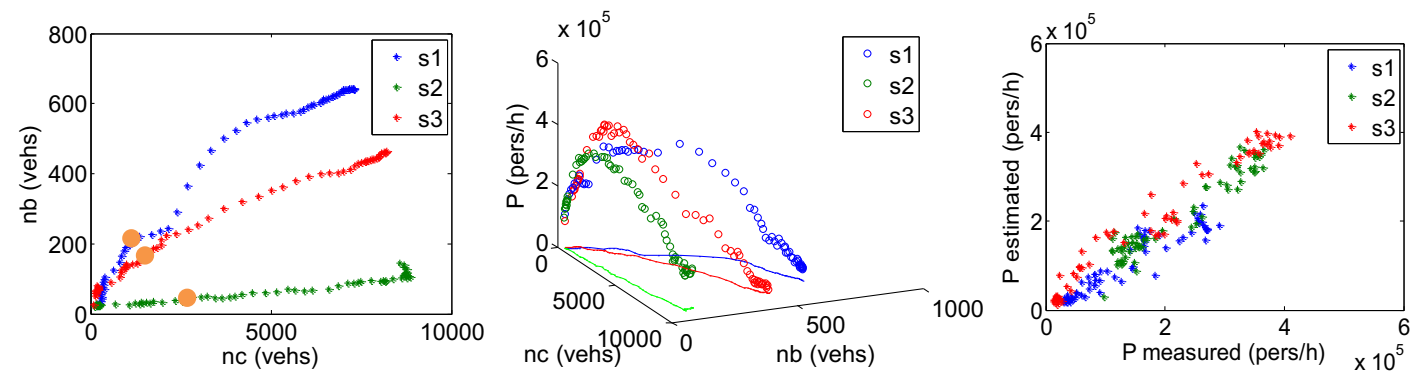

Fig. 10. Comparison of (a) (left) $n_{c}$ vs. $n_{b}$ for the entire network; (b) (middle) the estimated 3D-pMFD; and (c) (right) the measured $P$ vs. the estimated $P$ by model (13) for the three scenarios.

Fig. 10(c) compares the estimated $P$ from the model with the measured $P$ from the simulation for the whole network, clearly showing that with partitioning $P$ can be obtained more accurately. Furthermore by comparing the estimated passenger flow with and without partitioning, we find that the mean absolute error has a 10\% improvement (the higher the value of $\delta$, the higher the improvement).

\section{Discussion}

In this paper, we extended the single-mode network MFD model to a bi-modal three-dimensional MFD, with the consideration of passenger flows in addition to vehicle flows. We investigated the relation between the accumulation of cars and buses, and circulating flow of vehicles and passengers. We have shown via simulation experiments that: (i) an exponentialfamily function fits well the data points, (ii) the network's vehicle circulating flow decreases monotonically with the number of buses serving in the network, while the passenger throughput is maximized at a non-zero accumulation of buses. Then, we investigated the derivation of the passenger-flow 3D-MFD with an elegant analytical model. Furthermore, we showed that by applying a partitioning algorithm, which clusters a center and an outside region of the network according to the similarity of the car/bus density ratio, the passenger 3D-MFD model was able to reproduce the observed flow pattern from the simulated data. 
The findings of this paper are promising because the concept of a 3D-MFD can be used: (i) to monitor traffic performance in bi-modal networks, and traffic flow dynamics can be predicted given the current state of the two modes, (ii) to develop simple control policies in such a way that maximizes the bi-modal network vehicle or passenger throughput as in Ampountolas et al. (2014) and (iii) to identify different control strategies for different regions of a heterogeneous bi-modal urban network. Furthermore, policy-makers can adjust management measures to operate a city at different mobility levels as expressed by the MFD in Fig. 3(b). An expansion in the bus fleet of a city associated with a congestion pricing scheme to facilitate demand shift from cars to buses can succeed these goals. Further research is needed towards this direction. Ongoing work investigates (i) how perimeter control of cars can improve the system operation and decrease total passenger delays, and (ii) how optimal coordinated control strategies for different regions of a network with different multimodal characteristics can be developed.

Preferential treatment of buses in terms of control strategies should be another research priority. Transit priority strategies for mixed usage lanes and dedicated bus lanes should be carefully investigated. TSP can be more impactful, especially in case of high frequencies of buses. TSP can also influence the shape of the MFD and a methodology to identify critical intersections should be identified to avoid a negative network effect. Typically TSP is activated by the presence of the transit vehicle at certain time periods. Moreover, predicting aggregate behavior of a large number of public transit (PT) lines is easier than predicting the specific behavior/effect of one PT line and TSP. The results of this paper can also facilitate decisions about the redistribution of urban space among different modes as in Zheng and Geroliminis (2013).

\section{Acknowledgement}

This research was financially supported by the Swiss National Science Foundation (SNSF) Grant \# 200021-132501. The authors thank Yuxuan Ji, PhD student at Urban Transport Systems Laboratory, (LUTS-EPFL) for providing the partitioning algorithm.

\section{References}

Aboudolas, K., Geroliminis, N., 2013. Perimeter and boundary control in multi-reservoir heterogeneous networks. Transp. Res. Part B 55, $265-281$.

Ampountolas, K., Zheng, N., Geroliminis, N., 2014. Perimeter flow control for bi-modal urban road networks. In: The 93rd Annual Meeting of Transportation Research Board, Washington D.C., USA, 12-16 January 2014.

Boyaci, B., Geroliminis, N., 2011. Estimation of the network capacity for multimodal urban systems. Procedia - Soc. Behaviroal Sci. 16 , $803-813$.

Buisson, C., Ladier, C., 2009. Exploring the impact of homogeneity of traffic measurements on the existence of macroscopic fundamental diagrams. Transp. Res. Rec. 2124, 127-136.

Bureau of Public Roads, 1964. Traffic Assignment Manual. U.S. Department of Commerce, Urban Planning Division, Washington D.C., USA.

Christofa, E., Aboudolas, K., Skabardonis, A., 2013. Arterial traffic signal optimization: a person-based approach. In: The 92nd Annual Meeting of the Transportation Research Board, Washington D.C., USA, 13-17 January 2013.

Daganzo, C., 2007. Urban gridlock: macroscopic modeling and mitigation approaches. Transp. Res. Part B 41 (1), 49-62.

Daganzo, C., Cassidy, M., 2008. Effects of high occupancy vehicle lanes on freeway congestion. Transp. Res. Part B 42 (10), $861-872$.

Daganzo, C., Geroliminis, N., 2008. An analytical approximation for the macroscopic fundamental diagram of urban traffic. Transp. Res. Part B 42 (9), $771-$ 781.

De Berg, M., Cheong, O., van Kreveld, M., Overmars, M. (Eds.), 2008. Delaunay Triangulations. Computational Geometry: Algorithms and Applications, third ed. Springer-Verlag.

Edie, L., 1963. Discussion of traffic stream measurements and definitions. In: Proceedings of the 2nd International Symposium on the Theory of Traffic Flow, Paris, France, pp. 139-154, 25-27 July 1963.

Eichler, M., Daganzo, C., 2006. Bus lanes with intermittent priority: strategy formulae and an evaluation. Transp. Res. Part B 40 (9), $731-744$.

Gayah, V., Daganzo, C., 2011. Clockwise hysteresis loops in the macroscopic fundamental diagram: an effect of network instability. Transp. Res. Part B 45 (4), $643-655$.

Geroliminis, N., Boyaci, B., 2012. The effect of variability of urban systems characteristics in the network capacity. Transp. Res. Part B 46 (10), 1607-1623.

Geroliminis, N., Daganzo, C., 2008. Existence of urban-scale macroscopic fundamental diagrams: some experimental findings. Transp. Res. Part B 42 (9), 759-770.

Geroliminis, N., Sun, J., 2011. Properties of a well-defined macroscopic fundamental diagram for urban traffic. Transp. Res. Part B 45 (3), 605-617.

Godfrey, J., 1969. The mechanism of a road network. Traffic Eng. Control 11 (7), 323-327.

Gonzales, E., Daganzo, C., 2012. Morning commute with competing modes and distributed demand: user equilibrium, system optimum, and pricing. Transp. Res. Part B 46 (10), 1519-1534.

Gonzales, E., Chavis, C., Li, Y., Daganzo, C., 2011. Multimodal transport in Nairobi, Kenya: insights and recommendations with a macroscopic evidence-based model. In: The 90th Annual Meeting of the Transportation Research Board, Washington D.C., USA, $23-27$ January 2011.

Haddad, J., Geroliminis, N., 2012. On the stability of traffic perimeter control in two-region urban cities. Transp. Res. Part B 46 (9), $1159-1176$.

Haddad, J., Ramezani, M., Geroliminis, N., 2013. Cooperative traffic control of a mixed network with two urban regions and a freeway. Transp. Res. Part B Meth. 54, 17-36

Ji, Y., Geroliminis, N., 2012. On the spatial partitioning of urban transportation network. Transp. Res. Part B 46 (10), $1639-1656$.

Ji, Y., Daamen, W., Hoogendoorn, S., Hoogendoorn-Lanser, S., Qian, X., 2010. Macroscopic fundamental diagram: investigating its shape using simulation data. Transp. Res. Rec. 2161, 42-48.

Keyvan-Ekbatani, M., Kouvelas, A., Papamichail, I., Papageorgiou, M., 2012. Exploiting the fundamental diagram of urban network for feedback-based gating. Transp. Res. Part B 46 (10), 1393-1403.

Knoop, V., Hoogendoorn, S., van Lint, H., 2013. The impact of traffic dynamics on the macroscopic fundamental diagram. In: The 92nd Annual Meeting of the Transportation Research Board, Washington D.C., USA, 13-17 January 2013.

Li, J., Song, M., Zhang, W., 2010. Planning for bus rapid transit in single dedicated bus lane. Transp. Res. Rec. $2111,76-82$.

Leclercq, L., Geroliminis, N., 2013. Estimating MFDs in simple networks with route choice. Transp. Res. Part B 57, $468-484$.

Mahmassani, H., Williams, J., Herman, R., 1987. Performance of urban traffic networks. In: Proceedings of the 10th International Symposium on Transportation and Traffic Theory, Massachusetts, USA, pp. 1-20, 8-10 July 1987.

Mahmassani, H., Saberi, M., Zockaie, A., 2013. Urban network gridlock: theory, characteristics, and dynamics. Transp. Res. Part C 36, $480-497$. 
Mazloumian, A., Geroliminis, N., Helbing, D., 2010. The spatial variability of vehicle densities as determinant of urban network capacity. Philos. Trans. Roy. Soc. A 368 (1928), 4627-4648.

Mesbah, M., Currie, G., 2011. Optimization of transit priority in the transportation network using a genetic algorithm. IEEE Trans. Intell. Transp. Syst. 12 (3), 908-919.

Saberi, M., Mahmassani, H., 2012. Exploring properties of network-wide flow-density relations in a freeway network. Transp. Res. Rec. 2315, 153-163.

Shi, J., Malik, J., 2000. Normalized cuts and image segmentation. IEEE Trans. Pattern Anal. Mach. Intell. 22 (8), $888-905$.

Site, P., Filippi, F., 1998. Service optimization for bus corridors with short-run strategies and variable vehicle size. Transp. Res. Part A 32 (1), 19-38.

Tirachini, A., Hensher, D., 2011. Bus congestion, optimal infrastructure investment and the choice of a fare collection system in dedicated bus corridors. Transp. Res. Part B 45 (5), 828-844.

Tuerprasert, K., Aswakul, C., 2010. Multiclass cell transmission model for heterogeneous mobility in general topology of road network. J. Intell. Transp. Syst.: Technol., Plann., Oper. 14 (2), 68-82.

Zheng, N., Geroliminis, N., 2013. On the distribution of urban road space for multimodal congested networks. Transp. Res. Part B 57, $326-341$.

Zheng, N., Waraich, R., Axhausen, K., Geroliminis, N., 2012. A dynamic cordon pricing scheme combining the macroscopic fundamental diagram and an agent-based traffic model. Transp. Res. Part A 46 (8), 1291-1303.

Zheng, N., Aboudolas, K., Geroliminis, N., 2013. A city-scale macroscopic fundamental diagram for mixed bi-modal urban traffic. In: Proceeding of the 16th IEEE Conference on Intelligent Transportation System, The Hague, The Netherland, pp. 1029-1034, October 6-9 2013. 